\title{
The Characterization of Defects in Multi-Layered Composite Materials by Thermal Tomography Methods
}

\author{
W. SWIDERSKI \\ Military Institute of Armament Technology, Zielonka, Poland
}

\begin{abstract}
The idea of thermal tomography is slicing a work piece for some in-depth layers which exhibit the distribution of thermal properties in materials by the analogy with X-ray tomography. Thermal tomography is based on the analysis of the surface temperature evolution which follows thermal stimulation of test samples. In this paper, some experimental results of applying the dynamic thermal tomography algorithm to a carbon fibre reinforced plastic sample are presented. The efficiency of few data processing methods is discussed.
\end{abstract}

PACS numbers: 07.57.-c, 07.05.Pj, 42.30.-d, 42.30.Wb, 42.72.Ai

\section{Introduction}

In nondestructive testing of composite materials, not only defect detection but also both defect identification and characterization are of interest. The defect identification typically results in classifying defects by some groups, such as delaminations, inclusions, cracks, etc., while defect characterization often means determining defect size and depth. In this aspect, any tomographic procedure can be considered as a type of a defect characterization which allows the identification of a detected defect within a particular sample layer. "Slicing" a material by layers is particularly important in the inspection of multi-layered composite materials where the knowledge of defect depths (layers) is important.

The very term "tomography", or "computer tomography" (CT), came from the medical diagnostics where it concerned the examination of body organs by scanning them with $\mathrm{X}$ rays and further using a computer data treatment for reconstructing a series of cross-sectional scans along particular coordinate axes. In reconstruction algorithms, perturbation methods are usually applied, thus leading to modification of accepted model parameters.

In publications issued in two last decades, the combination of the terms "infrared", "thermographic", "thermal" and "tomography" specifies a number of different techniques intended for reconstructing the internal structure of opaque solid objects by the analogy with the computed $\mathrm{X}$-ray tomography.

The emission IR thermographic tomography of semi-transparent gases and plasma, as well as the IR tomography of charge carriers in semiconductors, are the techniques which are most close to the classical computed tomography, i.e. they realize a principle of rectilinear propagation of information carriers, such as electromagnetic radiation and/or charged particles $[1,2]$. A concept of thermal tomography in the application to medical objects was proposed by Nowakowski [3].

The temperature inside biological tissues can be also measured directly by using microwave radiometry, firstly proposed by Barrett et al. in the detection of breast cancer [4].

A special technique intended for determining in-depth temperatures is the passive acoustic thermotomography proposed by Guliayev et al. and studied by Pasechnik et al. [5].

Primarily, the term "thermal tomography" appeared in the 1980s in the thermal wave studies. The contemporary methodology and instrumentation in this area is traced to the work of Rosenzwaig and Gersho [6]. The idea of dynamic thermal tomography (DTT) was suggested by Vavilov and Shiryaev in 1986 (Tomsk Polytechnic University, Russia) on the wave of an explosive interest to the tomographic presentation of sample structure by passing radiation and/or particles through materials $[7,8]$.

Thermal tomography means "slicing" a sample into different in-depth layers where the distribution of thermal properties can be visualized, by analogy with X-ray tomography. Thermal tomography is accomplished by analyzing the surface temperature evolution of a component to be inspected following an initial thermal stimulation. Such stimulation can be achieved by applying pulse heating with duration ranging from a few milliseconds for high-conductivity materials (metals) to a few seconds for low-conductivity materials (composites). It is important that thermal stimulation is nondestructive, which means that it does not damage a test material due to overheating. Dynamic temperature signals which appear on the surface of test samples are recorded with an infrared camera. Technically, any thermal tomography procedure is a part of the corresponding standard procedure of thermal nondestructive testing. In fact, a key point in performing thermal tomography is a data processing algorithm which is supposed to convert the so-called heat transit times into material layer coordinates. Such an algorithm is to be applied to a captured image sequence where defect indications evolve in time. The important feature of DTT is the necessity of choosing a "non-defect" reference point, in regard of which heat transit times are analyzed. 
Thermal tomography allows:

- slicing a test sample into different in-depth layers,

- decreasing the influence of clutter (surface noise) on thermogram quality,

- enhancing the reliability of defect detection,

- evaluating defect depth with a reasonable accuracy.

\section{DTT approaches}

In this study, two approaches to thermal tomography have been explored. The first approach is "classical", and it takes into consideration a reference point. The second approach can be called "no-reference tomography" because it does not require introducing any specific reference point. This can be done in two different ways. First, the temperature behavior in non-defect points can be modeled by using the solutions which are well known from the heat conduction theory. Second, the polynomial fitting of different orders can be applied to the evolution of each pixel function assuming that non-defect evolutions are "smooth" while defect evolutions reveal some perturbations which should appear in high-order polynomials. The idea of such tomography was suggested by Vavilov [9], and in this study we shall use it.

The experimental part of the work has been performed on a sample made of carbon fibre reinforced plastic (CFRP). The sample contained square Teflon inserts placed between different composite plies.

The both approaches mentioned above will be analyzed. The "classical" DTT algorithm involves the conversion of pixel-based values of heat transit times into layer coordinates. It follows from the theory of thermal nondestructive testing that the DTT procedure is possible only in a one-sided test where heat transit times, e.g. the best observation times $\tau_{\mathrm{m}}$, increase with increasing material depth. We consider this algorithm classical because the differential temperature signals and heat transit times are determined in regard to a reference point chosen by the user in an area which is considered sound $[9,10]$. Note that, in a two-sided test, defect depth weakly influences surface temperature signals, thus allowing no tomographic data treatment.

It is clear from mentioned above that DTT is based on the fact that defects located at different depths are characterized by different $\tau_{\mathrm{m}}$ values which can be also considered as specific delay times. If the corresponding time vs. depth calibration function is available, a distribution of $\tau_{\mathrm{m}}$ can be converted into the respective distribution of defect depths (or material layer coordinates). It has been demonstrated that the maximum number of layers, in which a sample can be "sliced", is about 5 . The detected layers adjacent to the surface can be fairly thin but the deeper is a layer the greater should be its thickness. Very deep layers cannot be detected because they produce low signals $\Delta T$ which are normally defined as the difference between each thermogram point $T(i, j)$ and a chosen reference (non-defect) point $T\left(i_{\text {nd }}, j_{\text {nd }}\right)$ :

$$
\Delta T(i, j, \tau)=T(i, j, \tau)-T\left(i_{\mathrm{nd}}, j_{\mathrm{nd}}, \tau\right),
$$

where $i, j$ are pixel coordinates on thermogram, and $\tau$ is the time.

The values of the temperature signal $\Delta T$ can be positive or negative depending on the nature of defects, their size, as well as on heating parameters. Obviously, $\Delta T$ becomes zero in all pixels which behave identically to a chosen reference point. Some special synthetic images, called "maxigrams" and "timegrams", are associated with the DTT algorithm.

A maxigram is a synthetic image which exhibits the distribution of maximum values of some chosen informative parameters. For example, $\Delta T_{\mathrm{m}}$-maxigrams show all pixels in their "best" appearance in respect of a chosen reference point.

A timegram is a synthetic image which exhibits the distribution of heat transit times adhered to analyzed signal evolutions. For example, $\tau_{\mathrm{m}}$-timegrams show optimum observation times in each pixel in respect of a chosen reference point.

Any finite sequence of $\Delta T$ signals will reach the extremum value $\Delta T_{\mathrm{m}}$ at the time $\tau_{\mathrm{m}}$ called optimum observation time. The pixel-based pairs of $\Delta T_{\mathrm{m}}(i, j)$ and $\tau_{\mathrm{m}}(i, j)$ values produce positive or negative maxigrams ( $\Delta T_{\mathrm{m}}$-images) and timegrams $\left(\tau_{\mathrm{m}}\right.$-images) (Fig. 1) [10]. Maxigrams and timegrams are related to both defect depth and thickness, thus allowing defect characterization.
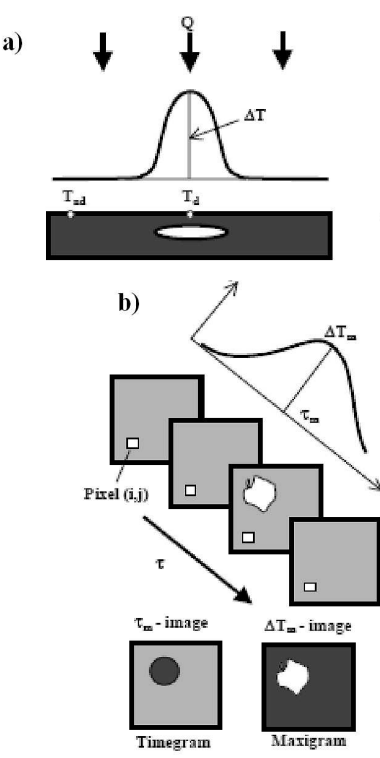

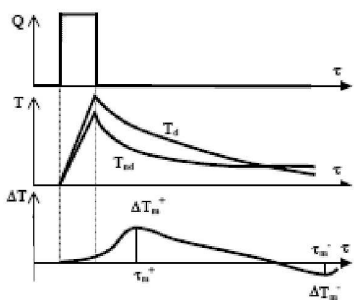

Fig. 1. Illustrating the principle of DTT: (a) location of points of interest and time evolution of surface temperature and the differential signal, (b) producing timegram and maxigram.

There are few formulae which describe the relationship between defect depth and optimum observation time. For 
instance, by using the solution for $1 \mathrm{D}$ heat conduction in a semi-infinite body after a Dirac heating pulse was applied, one can obtain that the observation time $\tau$ is a function (as the first approximation) of the squared depth $z[11]$ :

$$
\tau \approx \frac{z^{2}}{\alpha}
$$

where $\alpha$ is the thermal diffusivity of material, and $z$ is depth.

Thermal tomograms can be easily obtained by "truncating" respective timegrams. In other words, a tomogram is an image where all $\tau_{\mathrm{m}}$ pixel values are within a selected interval $\tau_{\mathrm{m} 1} \ldots \tau_{\mathrm{m} 2}$. The efficiency of the "classical" thermal tomography is related to how successfully a reference point is chosen. It depends on heating uniformity and efficiency of input data normalization.

The necessity of introducing a reference point is considered as the disadvantage of DTT because it involves the participation of an operator, even if any kind of quantitative assessment requires a sort of reference. In the case of DTT, it is possible to introduce a thermal model which would describe temperature evolutions in all sound points in a unique manner. Then, only significant deviations from such a model should be analyzed. This is the second approach accepted in this study. For example, a pixel temperature function can be described with two types of polynomials: of the high ("h") and low ("l") order [9]. It is supposed that the high-order polynomial describes subtle variations of the temperature functions in defect areas, while the low-order polynomial reflects only a general (non-defect) behavior of temperature. Then, the differential temperature signal

$$
\Delta T(i, j, \tau)=T^{(\mathrm{h})}(i, j, \tau)-T^{(\mathrm{l})}(i, j, \tau)
$$

may exhibit only defect signals which can be tomographically analyzed.

Equation (3) can be applied to each pixel without the necessity of choosing a reference point; therefore, we have called it "no reference" thermal tomography.

\section{Experimental setup}

The test sample having dimensions of $300 \times 300 \times 2 \mathrm{~mm}^{3}$ was made of 10-ply CFRP composite and contained 25 Teflon square-shaped inserts (lateral size $3,5,7,10$, and $15 \mathrm{~mm}$, thickness $0.1 \mathrm{~mm}$ ) which were placed between composite layers at various depths: $0.2,0.4,0.6,0.8$, and $1.0 \mathrm{~mm}$ (Fig. 2). The thermal properties of the materials have been assumed as follows: CFRP - thermal conductivity $\lambda=0.64 \mathrm{~W} /(\mathrm{m} \mathrm{K})$ (transverse) and $\lambda=1.28 \mathrm{~W} /(\mathrm{m} \mathrm{K})$ (lateral); thermal diffusivity $\alpha=$ $5.2 \times 10^{-7} \mathrm{~m}^{2} / \mathrm{s}$ and $10.4 \times 10^{-7} \mathrm{~m}^{2} / \mathrm{s}$, respectively; Teflon $-\lambda=0.23 \mathrm{~W} /(\mathrm{m} \mathrm{K}) ; \alpha=0.99 \times 10^{-7} \mathrm{~m}^{2} / \mathrm{s}$. The experimental results below have been obtained in Multipolar Infrared Vision Laboratory, Université Laval, Canada. The experimental setup consists of a heat source, an infrared camera and a computer system, thus enabling digital data recording in real time. The heat source consists of two flash tubes $3.2 \mathrm{~kJ}$ each (pulse duration $\approx 5 \mathrm{~ms}$ ).

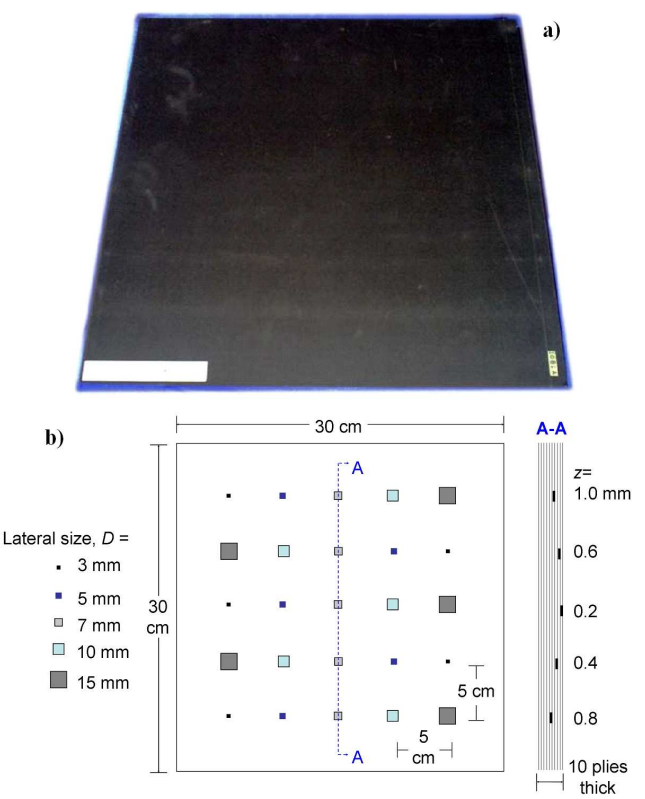

Fig. 2. CFRP sample $\left(300 \times 300 \times 2 \mathrm{~mm}^{3}\right)$ with 25 teflon inserts at different depths: (a) sample, (b) Teflon insert location.

The stage of sample cooling which followed flash stimulation was monitored on the same surface by using an FPA infrared camera (Santa Barbara SBF125), which operated in the spectral band from 3 to $5 \mu \mathrm{m}$. The tomographic analysis was applied to sequences of 250 images captured with a $90 \mathrm{~ms}$ acquisition interval. Due to the fact that the DTT algorithm can be applied only to a front-surface temperature response, only the one-sided test procedure was used in this study.

\section{Results}

\subsection{Optimum processing algorithm}

A source sequence has been processed by applying the algorithms known in pulsed thermal nondestructive testing and implemented in the ThermoLab software (Innovation, Ltd., Russia): normalization (N), polynomial fitting $(\mathrm{PF})$, pulse phase thermography (PPT), principal component analysis (PCA), correlation analysis (CA) and DTT. A detailed description of these algorithms is presented elsewhere [9]. It is worth noting that influence of the technical performance of IR imagers, such as temperature and spatial resolution, has not been explored in this study; these phenomena are believed to be of a secondary interest because the corresponding limiting parameters have never been reached in the experiments.

The signal-to-noise ratio (SNR) between a defect (see defect $\mathrm{A}$ in Fig. 3a, defect depth $1 \mathrm{~mm}$, defect size $15 \times 15 \mathrm{~mm}^{2}$ ) and neighbor non-defect areas have been chosen to compare the efficiency of the abovementioned processing algorithms. Note that the optimum source image (see Fig. 4a) is characterized by $\mathrm{SNR}=1$. Some 

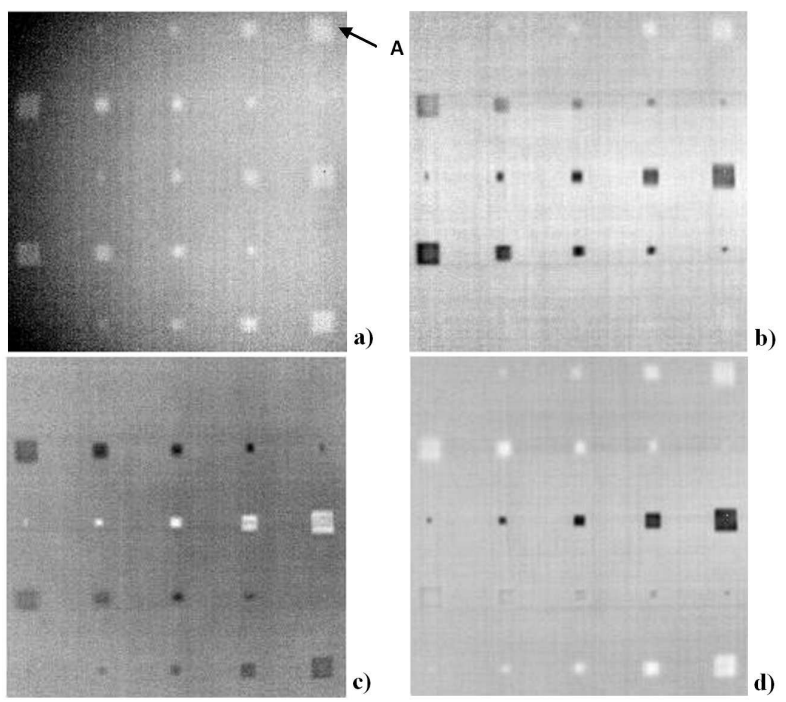

Fig. 3. Choosing an optimum data processing algorithm: (a) optimum source image after normalization $(\mathrm{SNR}=2.7)$, (b) phasegram $(\mathrm{SNR}=3.9),(\mathrm{c})$ polynomial coefficient $(\mathrm{SNR}=0.9)$, (d) 2nd principal component $(\mathrm{SNR}=9.5)$.

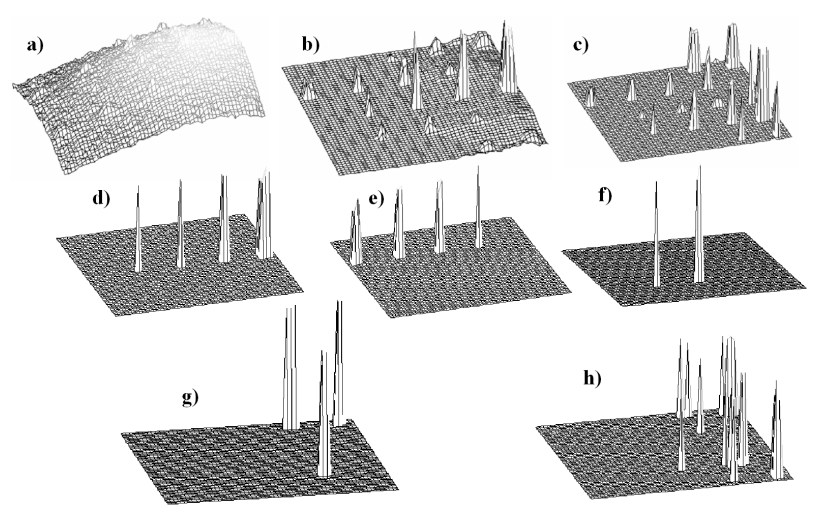

Fig. 4. Thermal tomography of the sample from Fig. 1: (a) best source image (SNR $=1.0)$, (b) maxigram, (c) timegram, (d) thermal tomogram (layer 0.24-0.31 mm), (e) thermal tomogram (layer $0.42-0.54 \mathrm{~mm}$ ), (f) thermal tomogram (layer $0.60-0.66 \mathrm{~mm}$ ), (g) thermal tomogram (layer $0.67-0.69 \mathrm{~mm})$, (h) thermal tomogram (layer $0.71-0.90 \mathrm{~mm}$ ).

processing results are shown in Fig. 3. The normalization significantly suppresses the phenomenon of uneven heating which is well seen in Fig. 4a, thus enhancing SNR to 2.7 (see Fig. 3a). The maximum value of $\mathrm{SNR}=9.5$ has been observed in the image of the 2nd principal component (PCA algorithm), see Fig. 3d. However, unlike the PPT algorithm which tends to improve the visibility of all defects, the PCA technique may enhance some defect indications at the expense of visibility of other defects. This PCA peculiarity is illustrated in Fig. 3d, where all defects located at the depth of $0.4 \mathrm{~mm}$ are scarcely seen. Also notice that, in the PCA image, the minimum detectable defect is that of size $5 \times 5 \mathrm{~mm}^{2}$ located at the depth of $1 \mathrm{~mm}$.

\subsection{Dynamic thermal tomography (classical approach)}

As mentioned above, the optimum source image shown in Fig. 4a reveals the pattern of uneven heating. Therefore, prior applying the DTT algorithm, the source data was normalized by the first image captured after the flash (Fig. 3a). Then, a reference point has been chosen close to defect $\mathrm{A}$ to produce the timegram shown in Fig. 4c. Respectively, it appears that the minimum detectable defect in the timegram is that of the size $10 \times 10 \mathrm{~mm}^{2}$ located at the depth of $1 \mathrm{~mm}$.

Thermal tomograms which represent the "slices" of the timegram are shown in Fig. $4 \mathrm{~d}-\mathrm{h}$. The calibration of the chosen in-depth layers depends on a value of CFRP thermal diffusivity inputted into the processing program (ThermoLab), which is similar to Eq. (2). The layer coordinates shown in Fig. $4 \mathrm{~d}-\mathrm{h}$ have been determined by the program automatically when assuming the CFRP thermal diffusivity being equal to $5.2 \times 10^{-7} \mathrm{~m}^{2} / \mathrm{s}$. The comparison between true depths of Teflon inserts in Fig. 1 and the tomographic data in Fig. 4 shows that the DTT algorithm allows a reasonably good match between the retrieved and true data (typically under 15-20\%).

It is interesting to note that the tomograms in Fig. $4 \mathrm{~d}-\mathrm{f}$ are "classical" in the sense that they exhibit only Teflon inserts located within the chosen layers. On the contrary, the images in Fig. $4 \mathrm{~g}$, $\mathrm{h}$ which are supposed to show the deeper layers (0.8 and $1 \mathrm{~mm}$ ) are different because, along with the defects within the chosen layers, they show the artifacts which come from the defects located in other layers. This phenomenon which is particularly well seen in Fig. 4h; it is related to the 3D nature of defect signals being discussed elsewhere $[9,10]$. Shortly, it is explained by the fact that each shallow sample layer is characterized not by a unique value of $\tau_{\mathrm{m}}$ but by a distribution of these values. On the contrary, longer $\tau_{\mathrm{m}}$ values correspond to deeper layers and thus appear in those layers. Therefore, when visualizing such deeper layers, one can see some "traces" of more shallow defects which appear as artifacts well seen in Fig. 4h. These artifacts have smaller differential amplitudes than principal defect areas, therefore, amplitude filtration can effectively suppress them but only in shallow layers, as in fact is shown in Fig. 4c-f. In deeper layers, these artifacts are inseparable from small defect signals.

\subsection{Dynamic thermal tomography ("no reference" approach)}

The same experimental data as described above has been processed by applying Eq. (3). First, the sequence was fitted with the 3rd and 6th order polynomials and the difference between two fitted sequences was analyzed. It has appeared that the experimental differential signals evolve in a random manner depending on the "quality" 
of polynomial fitting and their "slicing" is not a straightforward procedure. However, some preliminary results which are shown in Fig. 5 demonstrate that the visibility of defects within particular layers can be significantly enhanced in regard to the "classical" algorithm. Both tomograms in Fig. 5 show deeper defects without artifacts conditioned by other layers. However, the application of the "no reference" DTT algorithm still seems to be more the art rather than a well-defined procedure.

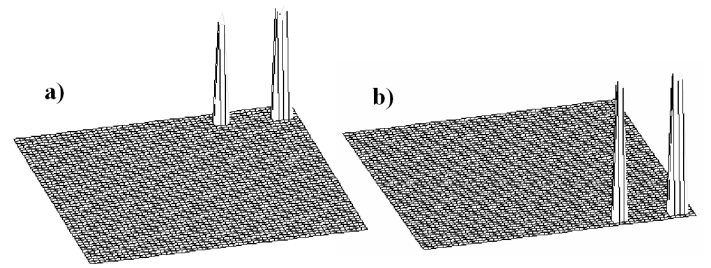

Fig. 5. "No-reference" thermal tomography algorithm: (a) thermal tomogram (layer 0.68-0.70 mm), (b) thermal tomogram (layer $0.71-0.90 \mathrm{~mm}$ ).

\section{Conclusions}

- In this study, two approaches to DTT have been applied in the inspection of a 10-ply (2 mm thick) CFRP composite sample which contained 25 Teflon inserts at depths up to $1 \mathrm{~mm}$.

- The first approach called "classical" is based on the analysis of heat transit times. It has allowed the detection of $10 \times 10 \mathrm{~mm}^{2}$ inserts at depths up to $1 \mathrm{~mm}$. The drawback of this approach, except the necessity of introducing a reference point, is the presence of artifacts which appear in a chosen sample layer due to the presence of defects in other layers. The artifacts can be effectively subdued in shallow layers by applying amplitude filtration, while in deeper layers the influence of more shallow layers worsens the quality of thermal tomograms.

- The second approach called "no reference" requires no reference point but involves the approximation of pixel-based temperature functions with polynomials of higher and lower orders. Low order polynomials reflect a non-defect behavior of test samples, while high order polynomials contain signal perturbations caused by defects. Therefore, the difference of two polynomials is supposed to exhibit the defects. Within this approach, it has been shown that, in some cases, the appearance of thermal tomograms can be improved but, in general, the results are unpredictable and require further research.

- The experimental results obtained on the CFRP sample have been processed by applying the algorithms of normalization, pulse phase thermography, polynomial fitting, PCA and CA. The best processing result appeared when using the PCA algorithm. This algorithm allowed the enhancement of the signal-to-noise ratio for the deepest defects from 1 (the optimum source image) to 9.5 (the image of the 2nd PCA component). However, the choice of a proper principal component depends very much on the preliminary knowledge about a tested sample and requires a certain experience from the thermographer.

- It is believed that the data processing techniques used in this study can be effectively applied in the inspection of composite and honeycomb structures which are increasingly used in the military and industry.

\section{Acknowledgments}

This study was supported by the Ministry of Science and Higher Education of Poland. The help of Prof. X. Maldague and Dr. C. Ibarra-Castaneda of Laval University, Canada, is thankfully appreciated.

\section{References}

[1] V.V. Pikalov, T.S. Melnikova, Plasma Tomography, Novosibirsk, Nauka, Moscow 1995, p. 210 (in Russian).

[2] S.S. Vorontsov, V.V. Pikalov, N.G. Preobrazhensky, N.V. Sharapova, The Algorithm for Projections Self-Matching in IR Thermographic Tomography. Automation of Aerodynamic Experiments, Institute of Theoretical and Applied Mechanics, Russian Academy of Sciences, Novosibirsk 1985, p. 63 (in Russian).

[3] A. Nowakowski, in: The Biomedical Engineering Handbook, Ed. J.D. Bronzino, 3rd ed., Medical Devices and Systems, Vol. 22-1, CRC Taylor \& Francis, London 2006, p. 22.

[4] A. Barrett, P.S. Myers, N.L. Sadowsky, Radio Sci. 12, 167 (1977).

[5] V.I. Pasechnik, A.A. Anosov, Yu.N. Barabanenkov, K.M. Bograchev, A.G. Selskiy, in: Proc. Nizhny Novgorod Acoustical Scientific Session, Universitet Nizhnego Novgoroda, Nizhny Novgorod 2002, p. 375 (in Russian)

[6] A. Rosencwaig, A. Gersho, Science 218, 223 (1982).

[7] V.P. Vavilov, V.V. Shiryaev, Thermal Tomograph, USSR Patent \# 1266308, 1984 (in Russian).

[8] X.P.V. Maldague, Theory and Practice of Infrared Technology for Nondestructive Testing, Wiley, New York 2001, p. 428.

[9] V. Vavilov, Thermal Nondestructive Testing, Handbook series: Nondestructive Testing, Vol. 5, Mashinostroyenie Publish., Moscow 2006, p. 380 (in Russian).

[10] V. Vavilov, Proc. SPIE "Thermosense-XII" 1313 , 178 (1990).

[11] P. Cielo, X. Maldague, A.A. Deom, R. Lewak, Mater. Evaluation 45, 452 (1987). 